\title{
Convergence of Numerical Solution of Generalized Theodorsen's Nonlinear Integral Equation
}

\author{
Mohamed M. S. Nasser ${ }^{1,2}$ \\ ${ }^{1}$ Department of Mathematics, Faculty of Science, King Khalid University, P.O. Box 9004, Abha, Saudi Arabia \\ ${ }^{2}$ Department of Mathematics, Faculty of Science, Ibb University, P.O. Box 70270, Ibb, Yemen
}

Correspondence should be addressed to Mohamed M. S. Nasser; mms_nasser@hotmail.com

Received 7 February 2014; Accepted 24 February 2014; Published 2 April 2014

Academic Editor: Samuel Krushkal

Copyright (C) 2014 Mohamed M. S. Nasser. This is an open access article distributed under the Creative Commons Attribution License, which permits unrestricted use, distribution, and reproduction in any medium, provided the original work is properly cited.

We consider a nonlinear integral equation which can be interpreted as a generalization of Theodorsen's nonlinear integral equation. This equation arises in computing the conformal mapping between simply connected regions. We present a numerical method for solving the integral equation and prove the uniform convergence of the numerical solution to the exact solution. Numerical results are given for illustration.

\section{Introduction}

Numerical methods for conformal mapping from a simply connected region onto another simply connected region are available only when one of the region is a standard region, mostly the unit disk $D$. Let $G$ and $\Omega$ be bounded simply connected regions in the $z$-plane and $w$-plane, respectively, such that their boundaries $\Gamma:=\partial G$ and $L:=\partial \Omega$ are smooth Jordan curves. Then the mapping $\Psi: G \rightarrow \Omega$ is calculated as the composition of the maps $G \rightarrow D \rightarrow \Omega$.

Recently, a numerical method has been proposed in [1] for direct approximation of the mapping $\Psi: G \rightarrow \Omega$. Assume that $\Gamma$ and $L$ are star-like with respect to the origin and defined by polar coordinates

$$
\eta(t)=\rho(t) e^{\mathrm{i} t}, \quad \zeta(t)=R(t) e^{\mathrm{i} t}, \quad 0 \leq t \leq 2 \pi,
$$

respectively, such that both $\rho$ and $R$ are $2 \pi$-periodic continuously differentiable positive real functions with nonvanishing derivatives. By the Riemann-mapping theorem, there exists a unique conformal mapping function $\Psi: G \rightarrow \Omega$ normalized by $\Psi(0)=0, \Psi^{\prime}(0)>0$. The boundary value of the function $\Psi$ is on the boundary $L$ and can be described as

$$
\Psi^{+}(\eta(t))=\zeta(S(t)), \quad 0 \leq t \leq 2 \pi,
$$

where $S(t)$ is the boundary correspondence function of the mapping function $\Psi$. The function $S(t)$ is a strictly increasing function so that $S(t)-t$ is a $2 \pi$-periodic function.

The function $S(t)$ is the unique solution of a nonlinear integral equation which can be interpreted as a generalization of Theodorsen's nonlinear integral equation [1]. The proof of the existence and the uniqueness of the solution of the nonlinear integral equation was given in [1] for regions $\Omega$ of which boundaries $L=\partial \Omega$ satisfy the so-called $\epsilon$-condition; that is,

$$
\varepsilon:=\max _{0 \leq t \leq 2 \pi}\left|\frac{R^{\prime}(t)}{R(t)}\right|<1
$$

In this paper, the nonlinear integral equation is solved by an iterative method. Each iteration of the iterative method requires solving an $n \times n$ linear system which is obtained by discretizing the integrals in the integral equation by the trapezoidal rule. The linear system is solved by a combination of the generalized minimal residual (GMRES) method and the fast multipole method (FMM) in $O(n \ln n)$ operations. The main objective of this paper is to prove the uniform convergence of the numerical solution to the exact solution. We also study the properties of the generalized conjugation operator. Numerical results are presented for illustration. 


\section{Auxiliary Materials}

2.1. The Functions $\theta$ and $\tau$. Let $w=f(z)$ be the mapping function from the simply connected region $G$ onto the unit disk $D$ with the normalization $f(0)=0$ and $f^{\prime}(0)>0$. Then the boundary value of the function $f$ is on the unit circle and can be described as

$$
f(\eta(t))=e^{\mathrm{i} \theta(t)}, \quad 0 \leq t \leq 2 \pi .
$$

The function $\theta(t)$ is the boundary correspondence function of the mapping function $f$ where $\theta(t)-t$ is a $2 \pi$-periodic function and $\theta^{\prime}(t)>0$ for all $t \in[0,2 \pi]$. Let $\tau(t)$ be the inverse of the function $\theta(t)$. Then $\tau(t)$ is the boundary correspondence function of the inverse mapping function $z=f^{-1}(w)$ from $D$ onto $G$; that is,

$$
f^{-1}\left(e^{\mathrm{i} t}\right)=\eta(\tau(t)), \quad 0 \leq t \leq 2 \pi,
$$

where $\tau(t)-t$ is a $2 \pi$-periodic function and $\tau^{\prime}(t)>0$ for all $t \in[0,2 \pi]$.

2.2. The Norms. Let $H$ be the space of all real Hölder continuous $2 \pi$-periodic functions on $[0,2 \pi]$. With the inner product

$$
(\gamma, \psi)=\frac{1}{2 \pi} \int_{0}^{2 \pi} \gamma(s) \psi(s) d s,
$$

the space $H$ is a pre-Hilbert space. We define the norm $\|\cdot\|_{2}$ by

$$
\|\gamma\|_{2}:=(\gamma, \gamma)^{1 / 2} .
$$

Since $s=\tau(t)$ and if $t=\theta(s)$, we have

$$
\begin{aligned}
\left\|\left(\theta^{\prime}\right)^{1 / 2} \gamma\right\|_{2} & =\int_{0}^{2 \pi} \theta^{\prime}(s) \gamma(s)^{2} d s \\
& =\int_{0}^{2 \pi} \gamma(\tau(t))^{2} d t=\|\gamma \circ \tau\|_{2} .
\end{aligned}
$$

With the norm $\|\cdot\|_{2}$, we define a norm $\|\cdot\|_{\theta}$ by

$$
\|\gamma\|_{\theta}:=\left\|\left(\theta^{\prime}\right)^{1 / 2} \gamma\right\|_{2}=\|\gamma \circ \tau\|_{2} .
$$

We define also the maximum norm $\|\cdot\|_{\theta}$ by

$$
\|\gamma\|_{\infty}:=\max _{0 \leq t \leq 2 \pi}|\gamma(t)| .
$$

Since $\theta(2 \pi)-\theta(0)=2 \pi$, we have

$$
\begin{aligned}
\|\gamma\|_{\theta}^{2} & =\frac{1}{2 \pi} \int_{0}^{2 \pi} \theta^{\prime}(t) \gamma^{2}(t) d t \\
& \leq\|\gamma\|_{\infty}^{2} \frac{1}{2 \pi} \int_{0}^{2 \pi} \theta^{\prime}(t) d t=\|\gamma\|_{\infty}^{2},
\end{aligned}
$$

which implies that

$$
\|\gamma\|_{\theta} \leq\|\gamma\|_{\infty} .
$$

Theorem 1. If $\int_{0}^{2 \pi} \theta^{\prime}(s) \gamma(s) d s=0$, then

$$
\|\gamma\|_{\infty}^{2} \leq 2 \pi\|\gamma\|_{\theta}\left\|\frac{\gamma^{\prime}}{\theta^{\prime}}\right\|_{\theta} .
$$

Proof. Since $s=\tau(t)$ and if $t=\theta(s)$, we have

$$
\begin{aligned}
\int_{0}^{2 \pi}(\gamma \circ \tau)(t) d t & =\int_{0}^{2 \pi} \gamma(\tau(t)) d t \\
& =\int_{0}^{2 \pi} \theta^{\prime}(s) \gamma(s) d s=0 .
\end{aligned}
$$

Thus, it follows from [2, page 68] that

$$
\|\gamma \circ \tau\|_{\infty}^{2} \leq 2 \pi\|\gamma \circ \tau\|_{2}\left\|(\gamma \circ \tau)^{\prime}\right\|_{2} .
$$

We have also

$$
\begin{aligned}
\left\|(\gamma \circ \tau)^{\prime}\right\|_{2}^{2} & =\frac{1}{2 \pi} \int_{0}^{2 \pi}\left|(\gamma \circ \tau)^{\prime}(t)\right|^{2} d t \\
& =\frac{1}{2 \pi} \int_{0}^{2 \pi} \gamma^{\prime}(\tau(t))^{2} \tau^{\prime}(t)^{2} d t \\
& =\frac{1}{2 \pi} \int_{0}^{2 \pi} \gamma^{\prime}(s)^{2} \frac{1}{\theta^{\prime}(s)} d s \\
& =\frac{1}{2 \pi} \int_{0}^{2 \pi} \theta^{\prime}(s)\left(\frac{\gamma^{\prime}(s)}{\theta^{\prime}(s)}\right)^{2} d s .
\end{aligned}
$$

Hence,

$$
\left\|(\gamma \circ \tau)^{\prime}\right\|_{2}^{2}=\left\|\frac{\gamma^{\prime}}{\theta^{\prime}}\right\|_{\theta}^{2} .
$$

Since $\tau(\cdot):[0,2 \pi] \rightarrow[0,2 \pi]$ is bijective, we have

$$
\begin{aligned}
\|\gamma \circ \tau\|_{\infty} & :=\max _{0 \leq t \leq 2 \pi}|\gamma(\tau(t))| \\
& =\max _{0 \leq \tau \leq 2 \pi}|\gamma(\tau)|=\|\gamma\|_{\infty} .
\end{aligned}
$$

Hence, (15) and (18) imply that

$$
\|\gamma\|_{\infty} \leq 2 \pi\|\gamma \circ \tau\|_{2}\left\|(\gamma \circ \tau)^{\prime}\right\|_{2} .
$$

Then (13) follows from (9), (17), and (19).

2.3. The Operators $\boldsymbol{K}$ and $\boldsymbol{J}$. The conjugation operator $\mathbf{K}$ is defined by

$$
\mathbf{K} \mu=\int_{0}^{2 \pi} \frac{1}{2 \pi} \cot \frac{s-t}{2} \mu(t) d t .
$$

Let $\mathbf{J}$ be the operator defined by

$$
\mathbf{J} \mu=\frac{1}{2 \pi} \int_{0}^{2 \pi} \mu(t) d t .
$$

Hence, the operators $\mathbf{K}$ and $\mathbf{J}$ satisfy [3]

$$
\mathbf{J K}=0, \quad \mathbf{K}^{2}=-\mathbf{I}+\mathbf{J} .
$$




\section{The Generalized Conjugation Operator}

Let $A$ be the complex $2 \pi$-periodic continuously differentiable function:

$$
A(s):=\eta(s) \text {. }
$$

We define the real kernels $M$ and $N$ as real and imaginary parts:

$$
M(s, t)+\mathrm{i} N(s, t):=\frac{1}{\pi} \frac{A(s)}{A(t)} \frac{\eta^{\prime}(t)}{\eta(t)-\eta(s)} .
$$

The kernel $N(s, t)$ is called the generalized Neumann kernel formed with $A$ and $\eta$. The kernel $N(s, t)$ is continuous and the kernel $M$ has the representation

$$
M(s, t)=-\frac{1}{2 \pi} \cot \frac{s-t}{2}+M_{1}(s, t),
$$

with a continuous kernel $M_{1}$. See [4] for more details.

We define the Fredholm integral operators $\mathbf{N}$ and $\mathbf{M}_{1}$ and the singular integral operator $\mathbf{M}$ on $H$ by

$$
\begin{aligned}
\mathbf{N} \mu & =\int_{0}^{2 \pi} N(s, t) \mu(t) d t, \\
\mathbf{M}_{1} \mu & =\int_{0}^{2 \pi} M_{1}(s, t) \mu(t) d t, \\
\mathbf{M} \mu & =\int_{0}^{2 \pi} M(s, t) \mu(t) d t .
\end{aligned}
$$

We define an operator $\mathbf{E}$ on $H$ by

$$
\mathbf{E}=-(\mathbf{I}-\mathbf{N})^{-1} \mathbf{M}
$$

The operator $\mathbf{E}$ is singular but bounded on $H$ [1]. Finally, we define an operator $\mathbf{J}_{\theta}$ by

$$
\mathbf{J}_{\theta} \mu=\frac{1}{2 \pi} \int_{0}^{2 \pi} \theta^{\prime}(t) \mu(t) d t
$$

Remark 2. When $\Gamma$ reduces to the unit, then $\theta^{\prime}(t)=1$, the operator $\mathbf{J}_{\theta}$ reduces to the operator $\mathbf{J}$, and the operator $\mathbf{E}$ reduces to the operator $\mathbf{K}$; that is, the operator $\mathbf{E}$ is a generalization of the well-known conjugation operator $\mathbf{K}$ (see [1] for more details).

The operator $\mathbf{E}$ is related to the operator $\mathbf{K}$ by [1]

$$
\mu=\mathbf{E} \gamma \quad \text { iff } \mu \circ \tau=\mathbf{K}(\gamma \circ \tau) .
$$

Since $\mu=(\mu \circ \theta) \circ \tau$ and $\gamma=(\gamma \circ \theta) \circ \tau$, it follows from (29) that

$$
\mu=\mathbf{K} \gamma \quad \text { iff } \mu \circ \theta=\mathbf{E}(\gamma \circ \theta) .
$$

Lemma 3 (see [1]). Let $\gamma, \mu \in H$ be given functions. Then $f(\eta(t))=\gamma(t)+i \mu(t)$ is the boundary value of an analytic function in $G$ with $\operatorname{Im} f(0)=0$ if and only if

$$
\mu=\mathbf{E} \gamma .
$$

Lemma 4 (see [1]). If $\gamma \in H$ and $\mu=\mathbf{E} \gamma$, then $\gamma=c-\mathbf{E} \mu$ with a real constant $c=f(0)$ where $f$ is the unique analytic function in $G$ with the boundary values $f(\eta(t))=\gamma(t)+i \mu(t)$ and $\operatorname{Im} f(0)=0$.

Lemma 5 (see [1]). The operator $\mathbf{E}$ has the following properties:

$$
\begin{aligned}
\operatorname{Null}(\mathbf{E}) & =\operatorname{span}\{1\}, \\
\mathbf{E}^{3} & =-\mathbf{E}, \\
\sigma(\mathbf{E}) & =\{0, \pm i\} .
\end{aligned}
$$

Lemma 6. The operator $\mathbf{E}$ has the norm

$$
\|\mathbf{E}\|_{\theta}=1 \text {. }
$$

Proof. The operator $\mathbf{E}$ has the norm $\|\mathbf{E}\|_{\theta} \leq 1$ [1]. Since i $\epsilon$ $\sigma(\mathbf{E})$, hence $1=|\mathbf{i}| \leq\|\mathbf{E}\|_{\theta} \leq 1$. Hence, we obtain (33).

Lemma 7. The operators $\mathbf{J}_{\theta}$ and $\mathbf{E}$ satisfy

$$
\mathbf{J}_{\theta} \mathbf{E}=0 \text {. }
$$

Proof. For any $\gamma \in H$, let $\mu=\mathbf{E} \gamma, \widehat{\mu}=\mu \circ \tau$, and $\widehat{\gamma}=\gamma \circ \tau$. Then, it follows from (29) that $\widehat{\mu}=\mathbf{K} \hat{\gamma}$. Thus,

$$
\begin{aligned}
\mathbf{J}_{\theta} \mathbf{E} \gamma(s) & =\mathbf{J}_{\theta} \mu(s) \\
& =\frac{1}{2 \pi} \int_{0}^{2 \pi} \theta^{\prime}(t) \mu(t) d t \\
& =\frac{1}{2 \pi} \int_{0}^{2 \pi} \mu(\tau(s)) d s \\
& =\frac{1}{2 \pi} \int_{0}^{2 \pi} \widehat{\mu}(s) d s \\
& =\mathbf{J} \widehat{\mu}(s) \\
& =\mathbf{J K} \hat{\gamma}(s),
\end{aligned}
$$

which by (22) implies that

$$
\mathbf{J}_{\theta} \mathbf{E} \gamma(s)=0 .
$$

Since (36) holds for all functions $\gamma \in H$, the operator identity (34) follows.

Lemma 8. The operator $\mathbf{E}$ satisfies

$$
\mathbf{E}^{2}=-\mathbf{I}+\mathbf{J}_{\theta}
$$

Proof. Let $\gamma \in H$ and $\mu=\mathbf{E} \gamma$. Then, by Lemma 4, $\gamma=c-\mathbf{E} \mu$ with a real constant $c$. By the definition of the operator $\mathbf{J}_{\theta}$, we have $\mathbf{J}_{\theta} c=c$. Since $\mathbf{J}_{\theta} \mathbf{E}=0$, we have

$$
\mathbf{J}_{\theta} \gamma=\mathbf{J}_{\theta} c-\mathbf{J}_{\theta} \mathbf{E}=c .
$$

Hence,

$$
\mathbf{E}^{2} \gamma=\mathbf{E} \mu=-\gamma+c=\left(-\mathbf{I}+\mathbf{J}_{\theta}\right) \gamma
$$

holds for all $\gamma \in H$. Thus, the operator identities (37) follow. 
Lemma 9. For all functions $\gamma \in H$, we have

$$
\|\mathbf{E} \gamma\|_{\theta} \leq\|\gamma\|_{\theta}
$$

with equality for all $\gamma$ with $\mathbf{J}_{\theta} \gamma=0$.

Proof. For all functions $\gamma \in H$, the inequality (40) follows from (33).

For all functions $\gamma$ with $\mathbf{J}_{\theta} \gamma=0$, we have from (37) that $\gamma=-\mathbf{E}^{2} \gamma$. Hence

$$
\|\gamma\|_{\theta}=\left\|\mathbf{E}^{2} \gamma\right\|_{\theta} \leq\|\mathbf{E} \gamma\|_{\theta} \leq\|\gamma\|_{\theta},
$$

which means that $\|\mathbf{E} \gamma\|_{\theta}=\|\gamma\|_{\theta}$.

Theorem 10. Let $\gamma \in H$ and $\mu=\mathbf{E} \gamma$. Then

$$
\frac{\mu^{\prime}}{\theta^{\prime}}=\mathbf{E}\left(\frac{\gamma^{\prime}}{\theta^{\prime}}\right) \text {. }
$$

Proof. For $\gamma \in H$ and $\mu=\mathbf{E} \gamma$, we have from (29) that $\mu \circ \tau=$ $\mathbf{K}(\gamma \circ \tau)$. Then, it follows from [2, page 64] that

$$
(\mu \circ \tau)^{\prime}=\mathbf{K}(\gamma \circ \tau)^{\prime} \text {. }
$$

Hence, by (30), we have

$$
(\mu \circ \tau)^{\prime} \circ \theta=\mathbf{E}\left((\gamma \circ \tau)^{\prime} \circ \theta\right)
$$

which implies that

$$
\frac{((\mu \circ \tau) \circ \theta)^{\prime}}{\theta^{\prime}}=\mathbf{E}\left(\frac{((\gamma \circ \tau) \circ \theta)^{\prime}}{\theta^{\prime}}\right) .
$$

Hence, we obtain (42).

\section{The Generalized Theodorsen Nonlinear Integral Equation}

The boundary correspondence function $S(t)$ is the unique solution of the nonlinear integral equation

$$
S(t)-t=\mathbf{E} \ln \frac{R(S(\cdot))}{\rho(\cdot)}(t)
$$

which is a generalization of the well-known Theodorsen integral equation [1]. Nonlinear integral equation (46) can be solved by the iterative method

$$
S_{k}(t)-t=\mathrm{E} \ln \frac{R\left(S_{k-1}(\cdot)\right)}{\rho(\cdot)}(t), \quad k=1,2,3, \ldots
$$

Then we have [1]

$$
\left\|S_{k}-S\right\|_{\theta} \leq \varepsilon^{k}\left\|S_{0}-S\right\|_{\theta} .
$$

Thus, if the curve $L$ satisfies the $\varepsilon$-condition (3), then

$$
\left\|S_{k}-S\right\|_{\theta} \longrightarrow 0
$$

That is, the approximate solutions $S_{k}(t)$ converge to $S(t)$ with respect to the norm $\|\cdot\|_{\theta}$ if $\varepsilon<1$.

In this section, we will prove the uniform convergence of the approximate solutions $S_{k}(t)$ to the exact solution $S(t)$. We will use the approach used in the proof of Proposition 1.5 in [2, page 69] related to Theodorsen's integral equation. See also $[5,6]$.

\section{Lemma 11. Consider}

$$
\mathbf{E}[\ln \rho(\cdot)](t)=t-\theta(t) .
$$

Proof. The function $\theta$ is the boundary correspondence function of the conformal mapping $f$ from $G$ onto the unit disk. Hence, the function $\theta(t)-t$ satisfies [1]

$$
\theta(t)-t=\mathbf{E} \ln \frac{1}{\rho(\cdot)}(t) .
$$

Then (50) follows from (51).

The previous lemma implies that (46) can be rewritten as

$$
S(t)-\theta(t)=\mathbf{E}[\ln R(S(\cdot))](t),
$$

and (47) can be rewritten as

$$
S_{k}(t)-\theta(t)=\mathbf{E}\left[\ln R\left(S_{k-1}(\cdot)\right)\right](t) .
$$

Thus

$$
S_{k}(t)-S(t)=\mathbf{E}\left[\ln R\left(S_{k-1}(\cdot)\right)-\ln R(S(\cdot))\right](t) .
$$

Lemma 12. Consider

$$
\left\|S_{k}-S\right\|_{\theta} \leq\left(\varepsilon+\left\|S_{0}-\theta\right\|_{\theta}\right) \varepsilon^{k} .
$$

Proof. Let $a$ be such that

$$
\frac{1}{1+\varepsilon} \leq \frac{R(t)}{a} \leq 1+\varepsilon, \quad \forall t .
$$

Then

$$
\left|\ln \frac{R(t)}{a}\right| \leq \ln (1+\varepsilon)<\varepsilon, \quad \forall t
$$

Hence,

$$
\left\|\ln \frac{R}{a}\right\|_{\infty}<\varepsilon
$$

Thus

$$
\begin{aligned}
\left\|\mathbf{E}\left[\ln \left(\frac{R(S(\cdot))}{a}\right)\right]\right\|_{\theta} & \left.\leq\|\mathbf{E}\|_{\theta} \| \ln \left(\frac{R(S)}{a}\right)\right] \|_{\theta} \\
& \left.\leq \| \ln \left(\frac{R(S)}{a}\right)\right] \|_{\infty}<\varepsilon .
\end{aligned}
$$

Since

$$
\begin{aligned}
S(t)-S_{0}(t) & =S(t)-\theta(t)+\theta(t)-S_{0}(t) \\
& =\mathbf{E}[\ln R(S(\cdot))](t)+\theta(t)-S_{0}(t)
\end{aligned}
$$


and $\mathrm{E}(\ln a)=0$, we have

$$
S(t)-S_{0}(t)=\mathbf{E}\left[\ln \left(\frac{R(S(\cdot))}{a}\right)\right](t)+\theta(t)-S_{0}(t),
$$

which implies that

$$
\begin{aligned}
\left\|S-S_{0}\right\|_{\theta} & \leq\left\|\mathbf{E}\left[\ln \left(\frac{R(S(\cdot))}{a}\right)\right]\right\|_{\theta} \\
& +\left\|\theta-S_{0}\right\|_{\theta} \leq \varepsilon+\left\|\theta-S_{0}\right\|_{\theta} .
\end{aligned}
$$

Hence (55) follows from (48).

Lemma 13. Consider

$$
\left\|\frac{S_{k}^{\prime}-S^{\prime}}{\theta^{\prime}}\right\|_{\theta} \leq \frac{\varepsilon}{\sqrt{1-\varepsilon^{2}}}\left(1+\left\|\frac{S_{0}^{\prime}}{\theta^{\prime}}\right\|_{\theta}\right) .
$$

Proof. We have

$$
\begin{aligned}
\left\|\frac{S_{k}^{\prime}-S^{\prime}}{\theta^{\prime}}\right\|_{\theta} & =\left\|\frac{S_{k}^{\prime}-\theta^{\prime}+\theta^{\prime}-S^{\prime}}{\theta^{\prime}}\right\|_{\theta} \\
& \leq\left\|\frac{S_{k}^{\prime}}{\theta^{\prime}}-1\right\|_{\theta}+\left\|\frac{S^{\prime}}{\theta^{\prime}}-1\right\|_{\theta} .
\end{aligned}
$$

Since

$$
\begin{aligned}
\left\|\frac{S^{\prime}}{\theta^{\prime}}-1\right\|_{\theta}^{2}= & \frac{1}{2 \pi} \int_{0}^{2 \pi} \theta^{\prime}(t)\left(\frac{S^{\prime}}{\theta^{\prime}}-1\right)^{2} d t \\
= & \frac{1}{2 \pi} \int_{0}^{2 \pi} \theta^{\prime}(t)\left(\frac{S^{\prime}}{\theta^{\prime}}\right)^{2} d t-2 \frac{1}{2 \pi} \int_{0}^{2 \pi} S^{\prime}(t) d t \\
& +\frac{1}{2 \pi} \int_{0}^{2 \pi} \theta^{\prime}(t) d t
\end{aligned}
$$

$\int_{0}^{2 \pi} S^{\prime}(t) d t=2 \pi$, and $\int_{0}^{2 \pi} \theta^{\prime}(t) d t=2 \pi$, we obtain

$$
\left\|\frac{S^{\prime}}{\theta^{\prime}}-1\right\|_{\theta}^{2}=\left\|\frac{S^{\prime}}{\theta^{\prime}}\right\|_{\theta}^{2}-1
$$

Similarly, we have

$$
\left\|\frac{S_{k}^{\prime}}{\theta^{\prime}}-1\right\|_{\theta}^{2}=\left\|\frac{S_{k}^{\prime}}{\theta^{\prime}}\right\|_{\theta}^{2}-1
$$

In view of Theorem 10, it follows from (52) and (53) that

$$
\begin{gathered}
\frac{S^{\prime}(t)}{\theta^{\prime}(t)}-1=\mathbf{E}\left[\frac{R^{\prime}(S(\cdot))}{R(S(\cdot))} \frac{S^{\prime}(\cdot)}{\theta^{\prime}(\cdot)}\right](t), \\
\frac{S_{k}^{\prime}(t)}{\theta^{\prime}(t)}-1=\mathbf{E}\left[\frac{R^{\prime}\left(S_{k-1}(\cdot)\right)}{R\left(S_{k-1}(\cdot)\right)} \frac{S_{k-1}^{\prime}(\cdot)}{\theta^{\prime}(\cdot)}\right](t) .
\end{gathered}
$$

Hence, it follows from (68) that

$$
\begin{aligned}
\left\|\frac{S^{\prime}}{\theta^{\prime}}-1\right\|_{\theta} & =\left\|\mathbf{E}\left[\frac{R^{\prime}(S(\cdot))}{R(S(\cdot))} \frac{S^{\prime}(\cdot)}{\theta^{\prime}(\cdot)}\right]\right\|_{\theta} \\
& \leq\|\mathrm{E}\|_{\theta}\left\|\frac{R^{\prime}(S(\cdot))}{R(S(\cdot))} \frac{S^{\prime}(\cdot)}{\theta^{\prime}(\cdot)}\right\|_{\theta} \leq \varepsilon\left\|\frac{S^{\prime}}{\theta^{\prime}}\right\|_{\theta} .
\end{aligned}
$$

By (70) and (66), we have

$$
\left\|\frac{S^{\prime}}{\theta^{\prime}}-1\right\|_{\theta}^{2} \leq \varepsilon^{2}\left\|\frac{S^{\prime}}{\theta^{\prime}}\right\|_{\theta}^{2}=\varepsilon^{2}+\varepsilon^{2}\left\|\frac{S^{\prime}}{\theta^{\prime}}-1\right\|_{\theta}^{2} .
$$

Hence,

$$
\left\|\frac{S^{\prime}}{\theta^{\prime}}-1\right\|_{\theta}^{2} \leq \frac{\varepsilon^{2}}{1-\varepsilon^{2}} .
$$

Similarly, it follows from (69) that

$$
\left\|\frac{S_{k}^{\prime}}{\theta^{\prime}}-1\right\|_{\theta} \leq \varepsilon\left\|\frac{S_{k-1}^{\prime}}{\theta^{\prime}}\right\|_{\theta}
$$

which by (67) implies that

$$
\begin{aligned}
\left\|\frac{S_{k}^{\prime}}{\theta^{\prime}}-1\right\|_{\theta}^{2} & \leq \varepsilon^{2}\left\|\frac{S_{k-1}^{\prime}}{\theta^{\prime}}\right\|_{\theta}^{2} \\
& =\varepsilon^{2}+\varepsilon^{2}\left\|\frac{S_{k-1}^{\prime}}{\theta^{\prime}}-1\right\|_{\theta}^{2} .
\end{aligned}
$$

Hence,

$$
\begin{aligned}
\left\|\frac{S_{k}^{\prime}}{\theta^{\prime}}-1\right\|_{\theta}^{2} & \leq \varepsilon^{2}+\varepsilon^{2}\left\|\frac{S_{k-1}^{\prime}}{\theta^{\prime}}-1\right\|_{\theta}^{2} \\
& \leq \cdots \leq \varepsilon^{2}+\varepsilon^{4}+\cdots+\varepsilon^{2 k}\left\|\frac{S_{0}^{\prime}}{\theta^{\prime}}-1\right\|_{\theta}^{2},
\end{aligned}
$$

which, in view of (67), implies that

$$
\left\|\frac{S_{k}^{\prime}}{\theta^{\prime}}-1\right\|_{\theta}^{2} \leq \frac{\varepsilon^{2}}{1-\varepsilon^{2}}\left(1+\left\|\frac{S_{0}^{\prime}}{\theta^{\prime}}-1\right\|_{\theta}^{2}\right)=\frac{\varepsilon^{2}}{1-\varepsilon^{2}}\left\|\frac{S_{0}^{\prime}}{\theta^{\prime}}\right\|_{\theta}^{2} .
$$

Then (63) follows from (64), (72), and (76).

Theorem 14. If $\varepsilon<1$, then the approximate solution $S_{k}$ converges uniformly to the exact solution $S$ with

$$
\begin{aligned}
\left\|S_{k}-S\right\|_{\infty} & \leq \sqrt{\frac{2 \pi}{\sqrt{1-\varepsilon^{2}}}} \\
& \times \sqrt{\left(\varepsilon+\left\|S_{0}-\theta\right\|_{\theta}\right)\left(1+\left\|\frac{S_{0}^{\prime}}{\theta^{\prime}}\right\|_{\theta}\right)} \varepsilon^{k / 2+1 / 2} .
\end{aligned}
$$

Proof. In view of (54), Lemma 7 implies that

$$
\int_{0}^{2 \pi} \theta^{\prime}(t)\left(S_{k}(t)-S(t)\right) d t=0
$$

Thus, we have from (13) that

$$
\left\|S_{k}-S\right\|_{\infty}^{2} \leq 2 \pi\left\|S_{k}-S\right\|_{\theta}\left\|\frac{S_{k}^{\prime}-S^{\prime}}{\theta^{\prime}}\right\|_{\theta} .
$$

Hence (77) follows from (55) and (63). 
The following corollary follows from the previous theorem.

Corollary 15. If

$$
S_{0}(t)=\theta(t)=t-\mathbf{E}[\ln \rho(\cdot)](t),
$$

then

$$
\left\|S_{k}-S\right\|_{\infty} \leq 2 \sqrt{\frac{\pi}{\sqrt{1-\varepsilon^{2}}}} \varepsilon^{k / 2+1} .
$$

Remark 16. When $\Gamma$ reduces to the unit, then

$$
\rho(t)=1, \quad \theta(t)=t, \quad \mathbf{E}=\mathbf{K} .
$$

Hence, the results presented in this section reduces to the results presented in [2] for Theodorsen's integral equation.

\section{Discretizing (47)}

In this paper, we will discretize (47) instead of (46). The numerical method used here is based on strict discretization of the integrals in the operator $\mathbf{E}$ by the trapezoidal rule which gives accurate results since the integrals are over $2 \pi$-periodic. Let $n$ be a given even positive integer. We define $n$ equidistant collocation points $s_{i}$ in the interval $[0,2 \pi]$ by

$$
t_{i}:=(i-1) \frac{2 \pi}{n}, \quad i=1,2, \ldots, n .
$$

Then, for $2 \pi$-periodic function $\gamma(t)$, the trapezoidal rule approximates the integral

$$
I=\int_{0}^{2 \pi} \gamma(t) d t
$$

by

$$
I_{n}=\frac{2 \pi}{n} \sum_{i=1}^{n} \gamma\left(t_{i}\right)
$$

If the function $\gamma(t)$ is continuous, then $\left|I-I_{n}\right| \rightarrow 0$. If the integrand $\gamma(t)$ is $k$ times continuously differentiable, then the rate of convergence of the trapezoidal rule is $O\left(1 / n^{k}\right)$. For analytic $\gamma(t)$, the rate of convergence is better than $O\left(1 / n^{k}\right)$ for any positive integer $k$ [7, page 83]. See also [8].

For $\gamma \in H$, the integral operator $\mathbf{N}$ will be discretized by the Nyström method as follows:

$$
\mathbf{N}_{n} \gamma(s)=\frac{2 \pi}{n} \sum_{j=1}^{n} N\left(s, t_{j}\right) \gamma\left(t_{j}\right)
$$

Hence, we have

$$
\begin{aligned}
& \left\|\left(\mathbf{N}-\mathbf{N}_{n}\right) \gamma\right\|_{\infty} \\
& \quad=\max _{s \in[0,2 \pi]}\left|\int_{0}^{2 \pi} N(s, t) \gamma(t) d t-\frac{2 \pi}{n} \sum_{j=1}^{n} N\left(s, t_{j}\right) \gamma\left(t_{j}\right)\right| .
\end{aligned}
$$

Since the kernel $N(s, t)$ is continuous on both variables and since the function $\gamma(t)$ is continuous, we have [9]

$$
\left\|\left(\mathbf{N}-\mathbf{N}_{n}\right) \gamma\right\|_{\infty} \longrightarrow 0
$$

The integral operator $\mathbf{M}_{1}$ will be discretized by the Nyström method as follows:

$$
\mathbf{M}_{1, n} \gamma(s)=\frac{2 \pi}{n} \sum_{j=1}^{n} M_{1}\left(s, t_{j}\right)\left[\gamma\left(t_{j}\right)-\gamma(s)\right] .
$$

Since the kernel $M_{1}(s, t)$ is continuous on both variables and since the function $\gamma(t)$ is continuous, we have [9]

$$
\begin{aligned}
\left\|\left(\mathbf{M}_{1}-\mathbf{N}_{1, n}\right) \gamma\right\|_{\infty} & \\
=\max _{s \in[0,2 \pi]} \mid & \int_{0}^{2 \pi} M_{1}(s, t) \gamma(t) d t \\
& \quad-\frac{2 \pi}{n} \sum_{j=1}^{n} M_{1}\left(s, t_{j}\right) \gamma\left(t_{j}\right) \mid \longrightarrow 0 .
\end{aligned}
$$

To discretize the operator $\mathbf{K} \gamma(s)$, we first approximate the function $\gamma(s)$ by the interpolating trigonometric polynomial of degree $n / 2$ which interpolates $\gamma(s)$ at the $n$ points $t_{j}, j=$ $1,2, \ldots, n$. That is,

$$
\gamma(s) \approx \sum_{i=0}^{n / 2} a_{i} \cos i s+\sum_{i=0}^{n / 2-1} b_{i} \sin i s .
$$

Then $\mathbf{K} \gamma(s)$ is approximated by

$$
\mathbf{K}_{n} \gamma(s)=\sum_{i=1}^{n / 2} a_{i} \sin i s-\sum_{i=0}^{n / 2-1} b_{i} \cos i s
$$

where [6]

$$
\left\|\left(\mathbf{K}-\mathbf{K}_{n}\right) \gamma\right\|_{\infty} \longrightarrow 0
$$

The integral operator $\mathbf{M}$ is then discretized by

$$
\mathbf{M}_{n}=\mathbf{M}_{1, n}-\mathbf{K}_{n} .
$$

Then, it follows from (90) and (93) that

$$
\left\|\left(\mathbf{M}-\mathbf{M}_{n}\right) \gamma\right\|_{\infty} \longrightarrow 0
$$

The operator $\mathbf{M}_{n}$ is bounded operator since the operator $\mathbf{M}_{1, n}$ is bounded $\left(M_{1}(s, t)\right.$ is continuous) and the operator $\mathbf{K}_{n}$ is bounded operator (see [6]).

Since the kernel $N(s, t)$ is continuous and $\lambda=1$ is not an eigenvalue of the kernel $N(s, t)[1]$, the operators $\mathbf{I}-\mathbf{N}_{n}$ are invertible and $\left(\mathbf{I}-\mathbf{N}_{n}\right)^{-1}$ are uniformly bounded for sufficiently large $n$ [9]. Hence, we discretize the operator $\mathbf{E}$ by the bounded operator

$$
\mathbf{E}_{n}:=-\left(\mathbf{I}-\mathbf{N}_{n}\right)^{-1} \mathbf{M}_{n} .
$$


Lemma 17. If $\gamma \in H$, then

$$
\left\|\left(\mathbf{E}-\mathbf{E}_{n}\right) \gamma\right\|_{\infty} \longrightarrow 0 .
$$

Proof. Let $\phi:=\mathbf{E} \gamma$ and $\phi_{n}:=\mathbf{E}_{n} \gamma$, then

$$
(\mathbf{I}-\mathbf{N}) \phi=-\mathbf{M} \gamma, \quad\left(\mathbf{I}-\mathbf{N}_{n}\right) \phi_{n}=-\mathbf{M}_{n} \gamma .
$$

Let also $\widehat{\phi}_{n}$ be the unique solution of the discretized equation

$$
\left(\mathbf{I}-\mathbf{N}_{n}\right) \widehat{\phi}_{n}=-\mathbf{M} \gamma
$$

Thus, we have

$$
\begin{aligned}
\left\|\left(\mathbf{E}-\mathbf{E}_{n}\right) \gamma\right\|_{\infty}= & \left\|\phi-\phi_{n}\right\|_{\infty} \leq\left\|\phi-\widehat{\phi}_{n}\right\|_{\infty} \\
& +\left\|\widehat{\phi}_{n}-\phi_{n}\right\|_{\infty} .
\end{aligned}
$$

Since the kernel $N$ is continuous and $\mathbf{N}_{n}$ is the discretization of $\mathbf{N}$, then it follows from [9, page 108] that

$$
\left\|\phi-\widehat{\phi}_{n}\right\|_{\infty} \longrightarrow 0 \text {. }
$$

Since $\left(\mathbf{I}-\mathbf{N}_{n}\right)^{-1}$ is bounded and $\gamma$ is continuous, then (95) implies that

$$
\begin{aligned}
\| \hat{\phi}_{n}- & \phi_{n} \|_{\infty} \\
& =\left\|\left(\mathbf{I}-\mathbf{N}_{n}\right)^{-1}\left(\mathbf{M}-\mathbf{M}_{n}\right) \gamma\right\|_{\infty} \\
& \leq\left\|\left(\mathbf{I}-\mathbf{N}_{n}\right)^{-1}\right\|_{\infty}\left\|\left(\mathbf{M}-\mathbf{M}_{n}\right) \gamma\right\|_{\infty} \longrightarrow 0,
\end{aligned}
$$

which with (100) and (101) implies (97).

To calculate the function $S_{k}$ in (47) for a given $S_{k-1}$, we replace the operator $\mathbf{E}$ in (47) by the approximate operator $\mathbf{E}_{n}$ to obtain

$$
S_{k, n}(s)-s=\mathbf{E}_{n} \ln \frac{R\left(S_{k-1}(\cdot)\right)}{\rho(\cdot)}(s),
$$

where $S_{k, n}$ is an approximation to $S_{k}$. Substituting $s=t_{i}$ and $i=1,2, \ldots, n$, in (103) we obtain

$$
S_{k, n}\left(t_{i}\right)-t_{i}=\mathbf{E}_{n} \ln \frac{R\left(S_{k-1}(\cdot)\right)}{\rho(\cdot)}\left(t_{i}\right), \quad i=1,2 \ldots, n .
$$

Equation (104) can be rewritten as

$$
\begin{array}{r}
\left(\mathbf{I}-\mathbf{N}_{n}\right)\left[S_{k, n}\left(t_{i}\right)-t_{i}\right]=-\mathbf{M}_{n} \ln \frac{R\left(S_{k-1}(\cdot)\right)}{\rho(\cdot)}\left(t_{i}\right), \\
i=1,2, \ldots, m,
\end{array}
$$

which represents an $n \times n$ linear system for the unknown $S_{k, n}\left(t_{1}\right), S_{k, n}\left(t_{2}\right), \ldots, S_{k, n}\left(t_{n}\right)$. By obtaining $S_{k, n}\left(t_{i}\right)$ for $i=$ $1,2 \ldots, n$, the function $S_{k, n}(s)$ can be calculated for $s \in$ $[0,2 \pi]$ by the Nyström interpolating formula. In the following lemma, we prove the uniform convergence of the approximate solution $S_{k, n}$ of discretized equation (103) to the solution $S_{k}$ of (46).
Lemma 18. Consider

$$
\left\|S_{k, n}-S_{k}\right\|_{\infty} \longrightarrow 0 \text { as } n \longrightarrow \infty .
$$

Proof. Let $\gamma(s):=\ln \left(R\left(S_{k-1}(s)\right)\right) / \rho(s)$. Then, we have

$$
S_{k, n}-S_{k}=\mathbf{E}_{n} \gamma-\mathbf{E} \gamma=\left(\mathbf{E}_{n}-\mathbf{E}\right) \gamma .
$$

Hence,

$$
\left\|S_{k, n}-S_{k}\right\|_{\infty} \leq\left\|\left(\mathbf{E}-\mathbf{E}_{n}\right) \gamma_{n}\right\|_{\infty} .
$$

The lemma is then followed from (97).

The proof of the uniform convergence of the approximate solution $S_{k, n}$ to the boundary correspondence function $S$ is given in the following theorem.

Theorem 19. If $\varepsilon<1$, then

$$
\left\|S_{k, n}-S\right\|_{\infty} \longrightarrow 0 \quad \text { as } k, n \longrightarrow \infty
$$

Proof. We have

$$
\left\|S_{k, n}-S\right\|_{\infty} \leq\left\|S_{k, n}-S_{k}\right\|_{\infty}+\left\|S_{k}-S\right\|_{\infty} .
$$

Since $\varepsilon<1$, it follows from (77) that $\left\|S_{k}-S\right\|_{\infty} \rightarrow 0$ as $k \rightarrow$ $\infty$. The theorem is then followed from (106).

\section{The Algebraic System}

Let $\mathbf{t}$ be the $n \times 1$ vector $\mathbf{t}:=\left(t_{1}, t_{2}, \ldots, t_{n}\right)^{T}$ where $T$ denotes transposition. Then, for any function $\gamma(t)$ defined on $[0,2 \pi]$, we define $\gamma(\mathbf{t})$ as the $n \times 1$ vector obtained by componentwise evaluation of the function $\gamma(t)$ at the points $t_{i}, i=1,2, \ldots, n$. As in MATLAB, for any two vectors $\mathbf{x}$ and $\mathbf{y}$, we define $\mathbf{x} * \mathbf{y}$ as the componentwise vector product of $\mathbf{x}$ and $\mathbf{y}$. If $\mathbf{y}_{j} \neq 0$, for all $j=1,2 \ldots,(m+1) n$, we define $\mathbf{x}$. $/ \mathbf{y}$ as the componentwise vector division of $\mathbf{x}$ by $\mathbf{y}$. For simplicity, we denote $\mathbf{x} * \mathbf{y}$ by $\mathbf{x y}$ and $\mathbf{x}$./y by $\mathbf{x} / \mathbf{y}$.

Let $\mathbf{x}_{k-1}=S_{k-1}(\mathbf{t})-\mathbf{t}$ (given) and $\mathbf{x}_{k}=S_{k, n}(\mathbf{t})-\mathbf{t}$ (unknown). Then system (105) can be rewritten as

$$
(I-B) \mathbf{x}_{k}=-C \ln \frac{R\left(\mathbf{x}_{k-1}+\mathbf{t}\right)}{\rho(\mathbf{t})}, \quad k=1,2, \ldots,
$$

where $I$ is the $n \times n$ identity matrix, $B$ is the discretized matrix of the operator $\mathbf{N}$, and $C$ is the discretized matrix of the operator $\mathbf{M}$ [1]. Linear system (111) is uniquely solvable $[4,10,11]$.

We start the iteration in (47) with $S_{k}(t)=t$ and iterate until $\left\|S_{k}-S_{k-1}\right\|_{\infty}<$ tol where tol is a given tolerance; that is, we start the iteration in (111) with $\mathbf{x}_{0}=\mathbf{0}$ and iterate until $\left\|\mathbf{x}_{k}-\mathbf{x}_{k-1}\right\|_{\infty}<$ tol. Each iteration in (111) requires solving a linear system for $\mathbf{x}_{k}$ given $\mathbf{x}_{k-1}$. Linear system (111) is solved in $O(n \ln n)$ operations by the fast method presented in [11, 12] which is based on a combination of the MATLAB function gmres and the MATLAB function zfmm2dpart in the MATLAB toolbox FMMLIB2D [13]. In the numerical results below, for 

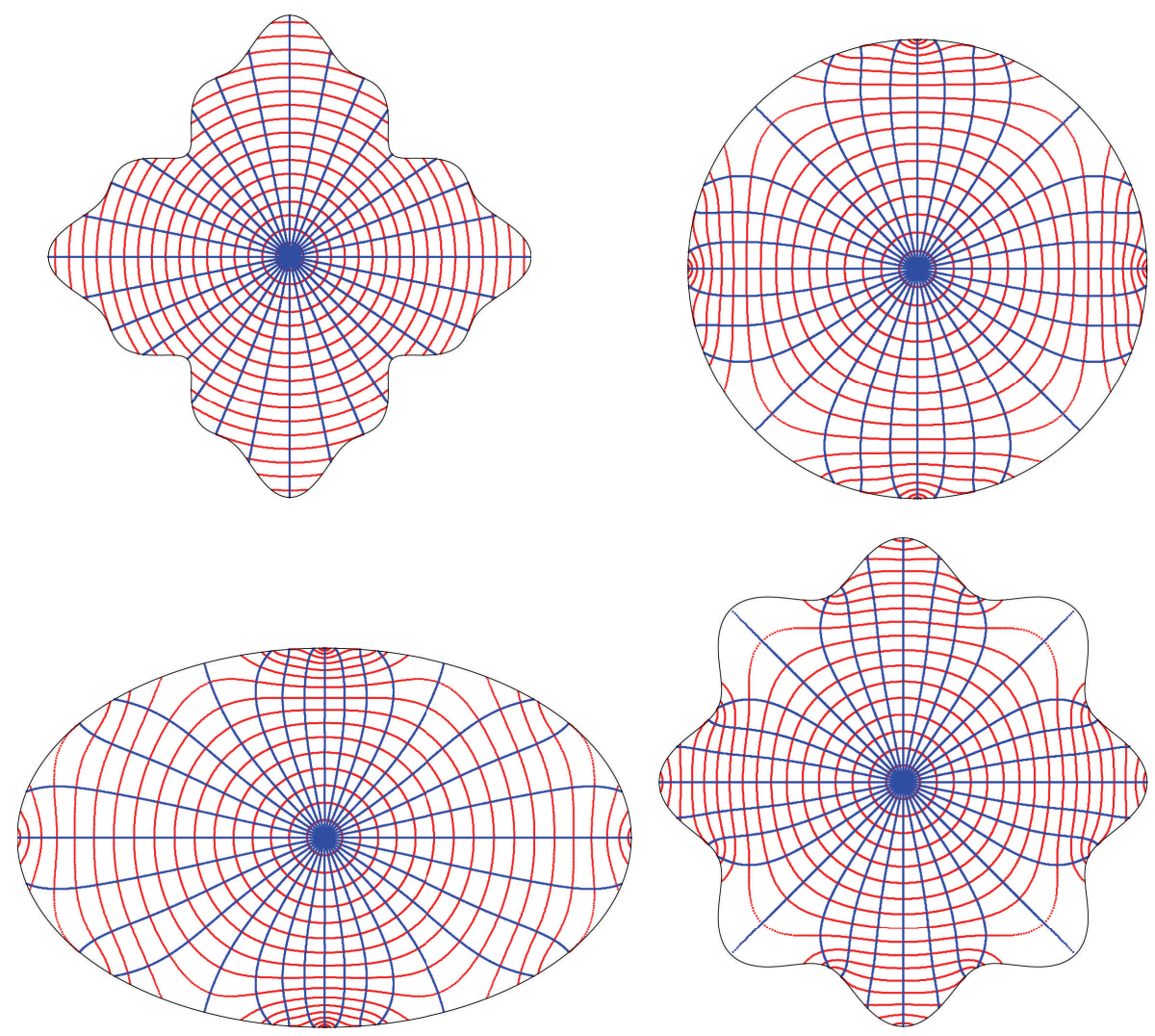

Figure 1: The conformal mappings from $G_{1}$ onto $\Omega_{1}, \Omega_{2}$, and $\Omega_{3}$.
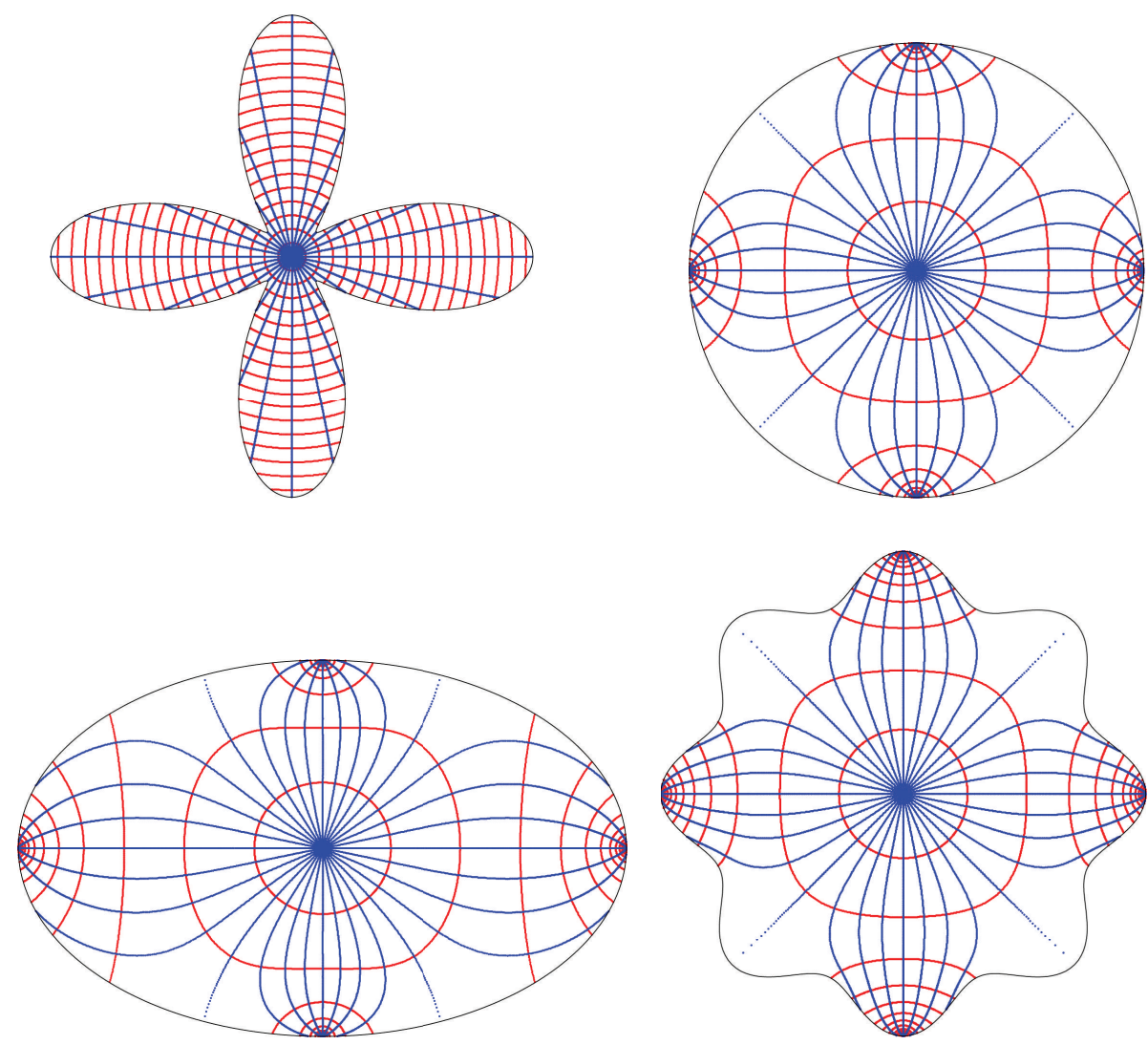

Figure 2: The conformal mappings from $G_{2}$ onto $\Omega_{1}, \Omega_{2}$, and $\Omega_{3}$. 

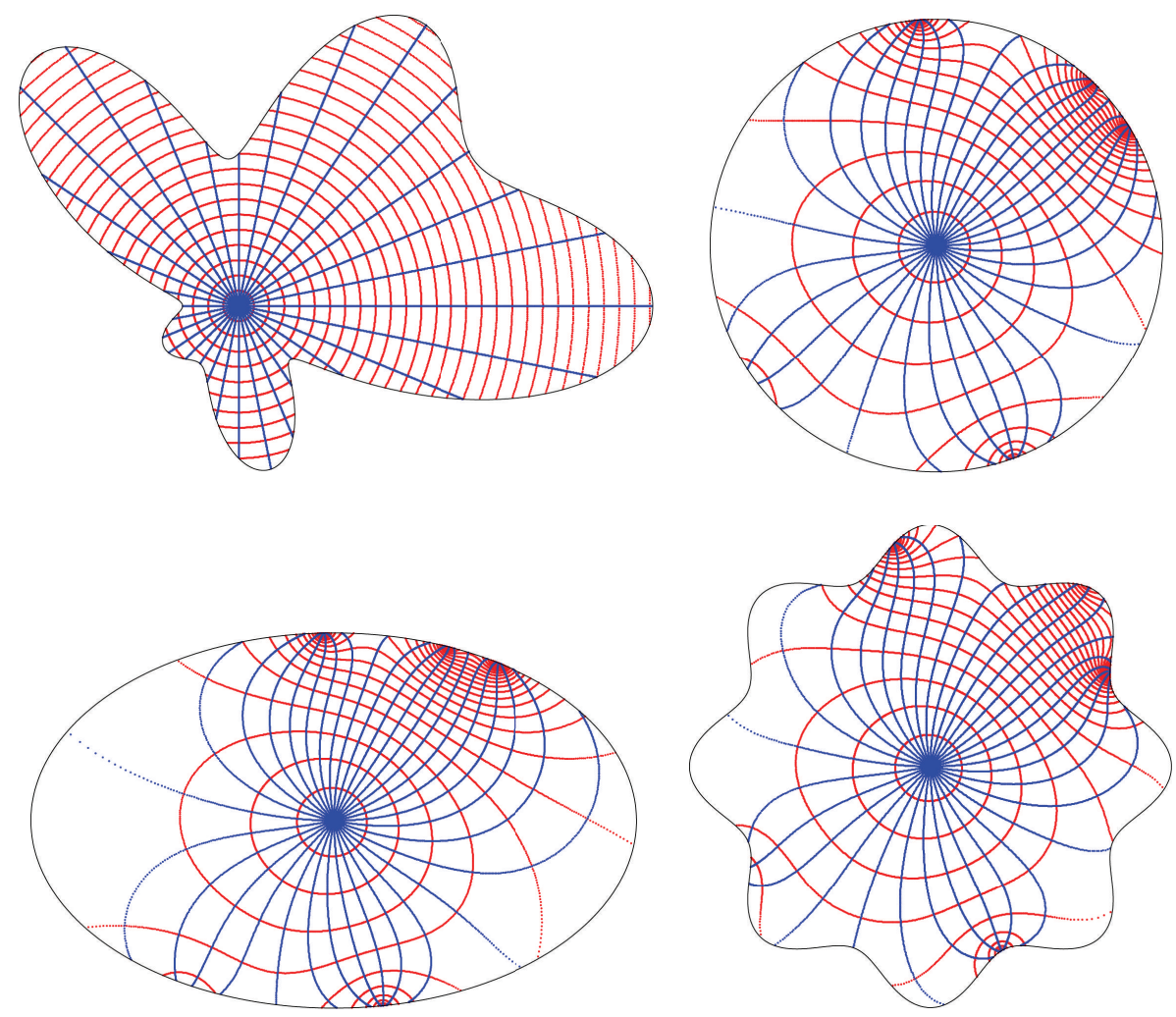

FIGURE 3: The conformal mappings from $G_{3}$ onto $\Omega_{1}, \Omega_{2}$, and $\Omega_{3}$.

function zfmm2dpart, we assume thatiprec $=4$ which means that the tolerance of the FMM is $0.5 \times 10^{-12}$. For the function gmres, we choose the parameters restart $=10$, gmrestol $=10^{-12}$, and maxit $=10$, which means that the GMRES method is restarted every 10 inner iterations, the tolerance of the GMRES method is $10^{-12}$, and the maximum number of outer iterations of GMRES method is 10 . See $[11,12]$ for more details.

By obtaining $\mathbf{x}_{k}$, we obtain the values $S_{k, n}\left(t_{i}\right)$ for $i=$ $1,2, \ldots, n$. Then, the function $S_{k, n}(s)-s$ can be calculated for $s \in[0,2 \pi]$ by the Nyström interpolating formula. The convergence of $S_{k, n}(s)$ to $S(s)$ follows from Theorem 19. Then the values of the mapping function $\Psi$ can be computed from (2). The interior values of the mapping function can be computed by the Cauchy integral formula which can be computed using the fast method presented in [12].

\section{Numerical Examples}

In this section, we will compute the conformal mapping from three simply connected regions $G_{1}, G_{2}$, and $G_{3}$ onto three simply connected regions $\Omega_{1}, \Omega_{2}$, and $\Omega_{3}$. The boundaries $\Gamma_{1}$, $\Gamma_{2}$, and $\Gamma_{3}$ of the regions $G_{1}, G_{2}$, and $G_{3}$ are parameterized by

$$
\eta(t)=\rho(t) e^{\mathrm{i} t}, \quad 0 \leq t \leq 2 \pi
$$

where the function $\rho(t)$ is given by

$$
\begin{gathered}
\Gamma_{1}: \rho(t)=1+\frac{1}{4} \cos ^{3} 4 t, \\
\Gamma_{2}: \rho(t)=1+\frac{3}{4} \cos 4 t, \\
\Gamma_{3}: \rho(t)=e^{\cos t} \cos ^{2} 2 t+e^{\sin t} \sin ^{2} 2 t .
\end{gathered}
$$

The boundaries $L_{1}, L_{2}$, and $L_{3}$ of the regions $\Omega_{1}, \Omega_{2}$, and $\Omega_{3}$ are parameterized by

$$
\eta(t)=R(t) e^{\mathrm{it}}, \quad 0 \leq t \leq 2 \pi
$$

where the function $R(t)$ is given by

$$
\begin{gathered}
L_{1}: R(t)=1, \\
L_{2}: R(t)=\frac{\alpha}{\sqrt{1-\left(1-\alpha^{2}\right) \cos ^{2} t}}, \\
\alpha=0.6180339630899485, \\
L_{3}: R(t)=1+\frac{1}{10} \cos 8 t .
\end{gathered}
$$




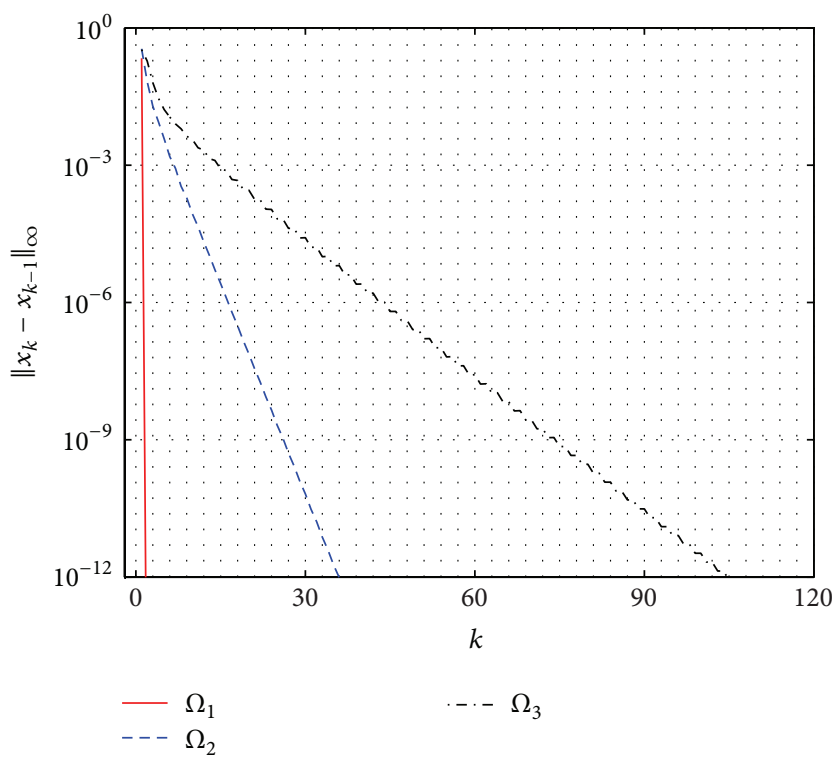

(a)

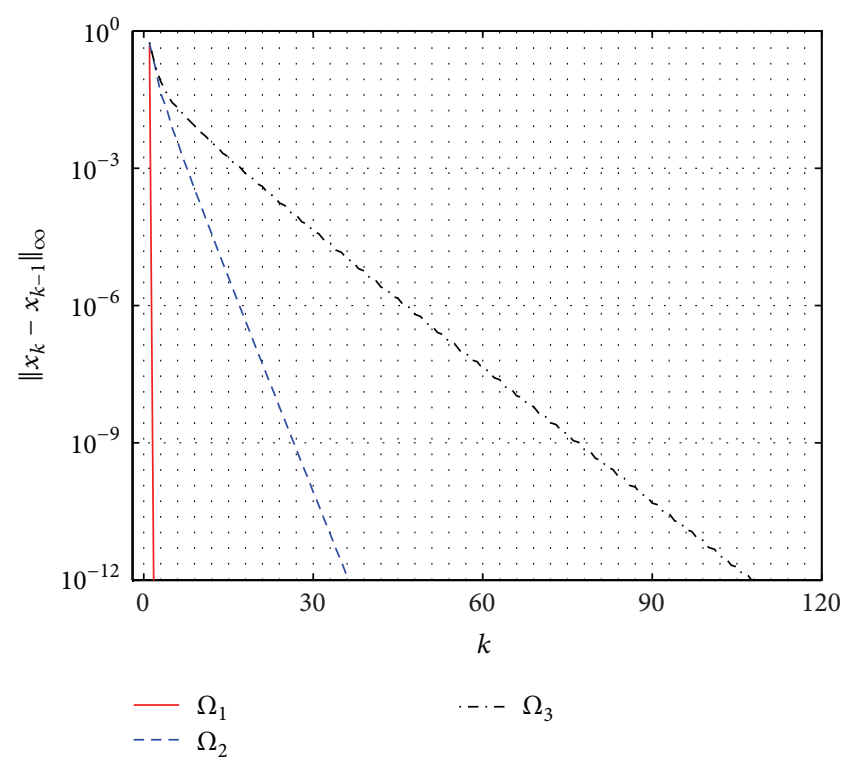

(b)

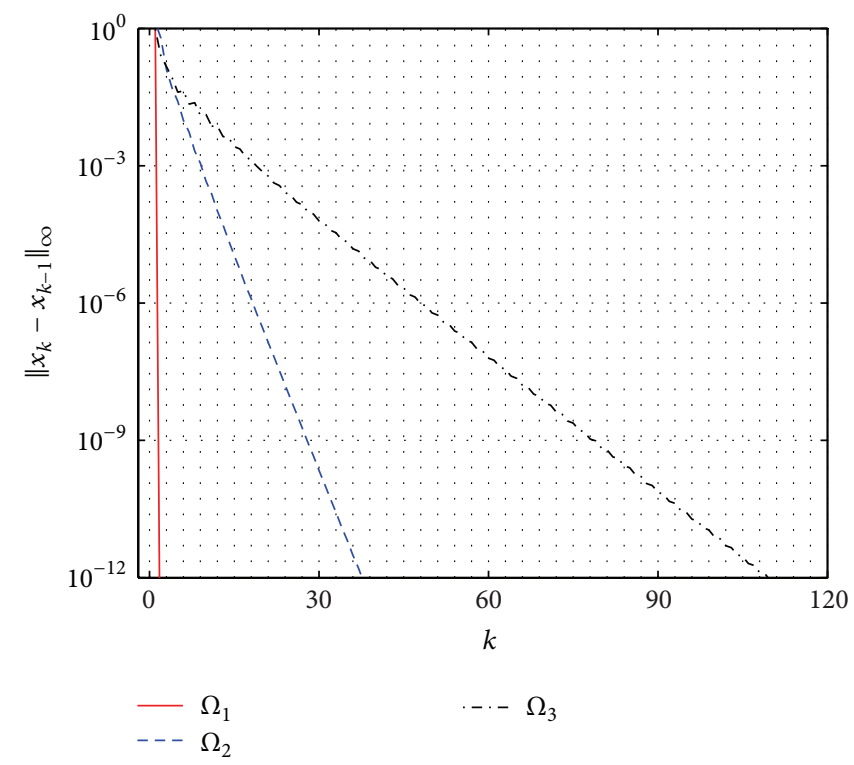

(c)

FIGURE 4: The error norm $\left\|\mathbf{x}_{k}-\mathbf{x}_{k-1}\right\|_{\infty}$ versus the iteration number $k$ for (a) the conformal mapping from $G_{1}$ onto $\Omega_{1}, \Omega_{2}$, and $\Omega_{3}$, (b) the conformal mapping from $G_{2}$ onto $\Omega_{1}, \Omega_{2}$, and $\Omega_{3}$, and (c) the conformal mapping from $G_{3}$ onto $\Omega_{1}, \Omega_{2}$, and $\Omega_{3}$.

The curves $L_{1}, L_{2}$, and $L_{3}$ satisfy the $\varepsilon$-condition where

$$
\begin{gathered}
L_{1}: \varepsilon=\left\|\frac{R^{\prime}(t)}{R(t)}\right\|_{\infty}=0, \\
L_{2}: \varepsilon=\left\|\frac{R^{\prime}(t)}{R(t)}\right\|_{\infty}=0.5<1, \\
L_{3}: \varepsilon=\left\|\frac{R^{\prime}(t)}{R(t)}\right\|_{\infty}=0.80403<1 .
\end{gathered}
$$

The numerical results obtained with $n=4096$ and tol $=10^{-12}$ are shown in Figures 1, 2, and 3. The error norm $\left\|\mathbf{x}_{k}-\mathbf{x}_{k-1}\right\|_{\infty}$ versus the iteration number $k$ in (111) is shown in Figure 4. It is clear from Figure 4 that the number of iterations in (111) depends only on the boundary $L$ of the image region. More precisely, it depends on $\varepsilon$. The iterations in (111) converge only if $\varepsilon<1$. For small $\varepsilon$, a few number of iterations are required for convergence. For values of $\varepsilon$ close to 1 , a large number of iterations are required for convergence.

\section{Conflict of Interests}

The authors declare that there is no conflict of interests regarding the publication of this paper. 


\section{References}

[1] M. M. S. Nasser, "A nonlinear integral equation for numerical conformal mapping," Advances in Pure and Applied Mathematics, vol. 1, no. 1, pp. 47-63, 2010.

[2] D. Gaier, Konstruktive Methoden der konformen Abbildung, Springer, Berlin, Germany, 1964.

[3] R. Wegmann, "Discretized versions of Newton type iterative methods for conformal mapping," Journal of Computational and Applied Mathematics, vol. 29, no. 2, pp. 207-224, 1990.

[4] R. Wegmann, A. H. M. Murid, and M. M. S. Nasser, "The Riemann-Hilbert problem and the generalized Neumann kernel," Journal of Computational and Applied Mathematics, vol. 182, no. 2, pp. 388-415, 2005.

[5] D. Gaier, "Numerical methods in conformal mapping," in Computational Aspects of Complex Analysis, K. E. Werner, L. Wuytack, and E. Ng, Eds., vol. 102, pp. 51-78, Reidel, Dordrecht, The Netherlands, 1983.

[6] R. Wegmann, "Methods for numerical conformal mapping," in Handbook of Complex Analysis: Geometric Function Theory, R. Kuhnau, Ed., vol. 2, pp. 351-477, Elsevier B. V., Amsterdam, The Netherlands, 2005.

[7] A. R. Krommer and C. W. Ueberhuber, Numerical Integration on Advanced Computer Systems, vol. 848, Springer, Berlin, Germany, 1994.

[8] P. J. Davis and P. Rabinowitz, Methods of Numerical Integration, Academic Press, New York, NY, USA, 2nd edition, 1984.

[9] K. E. Atkinson, The Numerical Solution of Integral Equations of the Second Kind, vol. 4, Cambridge University Press, Cambridge, UK, 1997.

[10] M. M. S. Nasser, "A boundary integral equation for conformal mapping of bounded multiply connected regions," Computational Methods and Function Theory, vol. 9, no. 1, pp. 127-143, 2009.

[11] M. M. S. Nasser, "Fast solution of boundary integral equations with thegeneralized Neumann kernel," http://arxiv.org/ abs/1308.5351.

[12] M. M. S. Nasser and F. A. A. Al-Shihri, "A fast boundary integral equation method for conformal mapping of multiply connected regions," SIAM Journal on Scientific Computing, vol. 35, no. 3, pp. A1736-A1760, 2013.

[13] L. Greengard and Z. Gimbutas, "FMMLIB2D: a MATLAB toolbox for fast multipole method in two dimensions," Version 1.2, 2012, http://www.cims.nyu.edu/cmcl/fmm2dlib/fmm2dlib .html. 


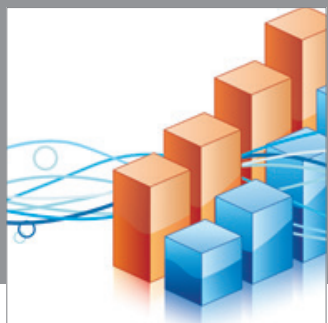

Advances in

Operations Research

mansans

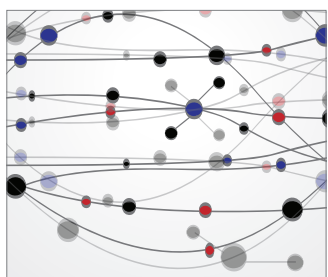

The Scientific World Journal
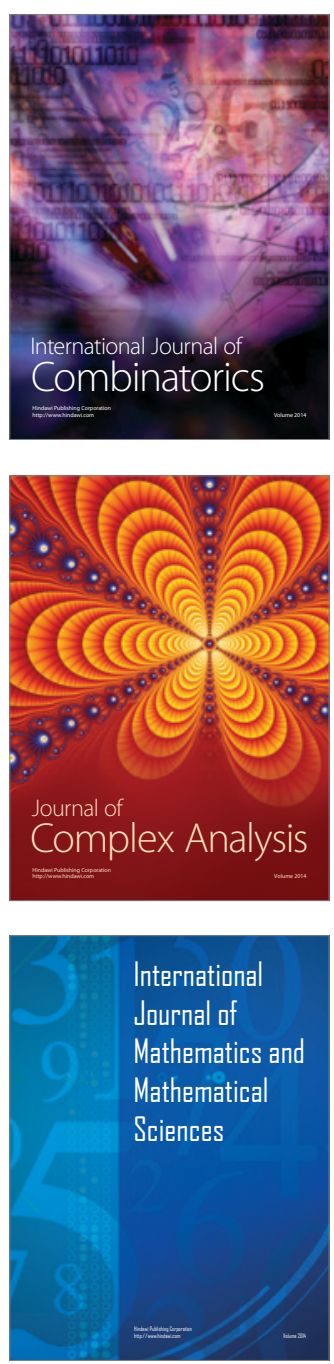
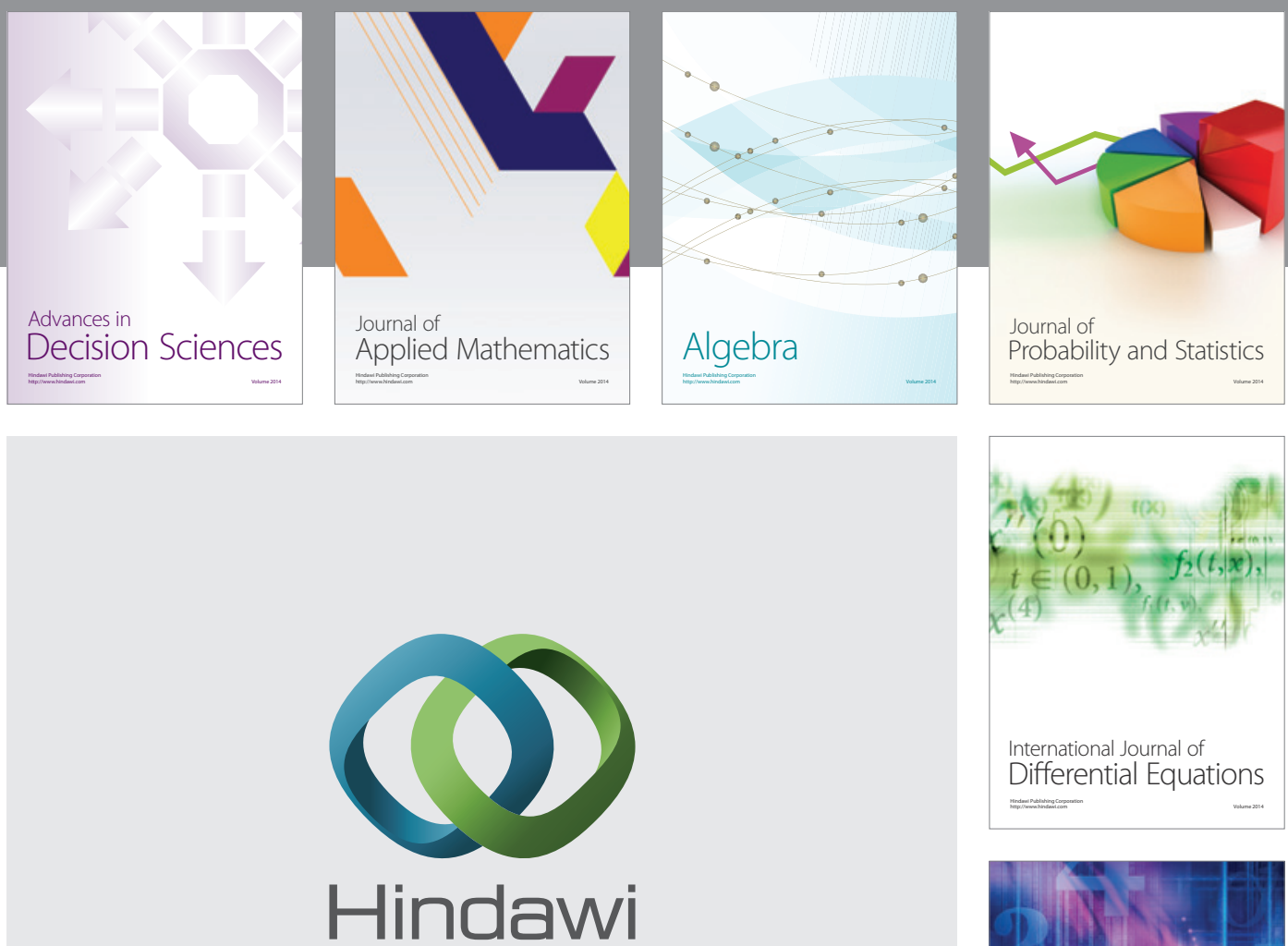

Submit your manuscripts at http://www.hindawi.com
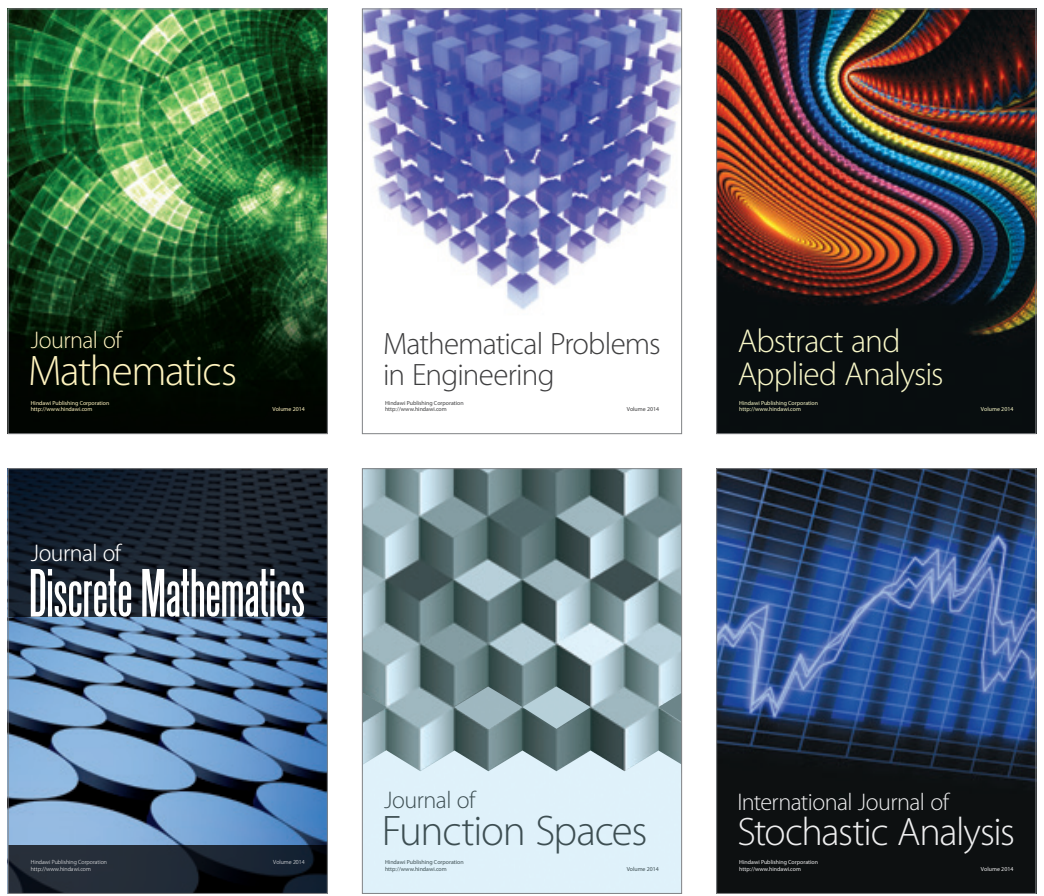

Journal of

Function Spaces

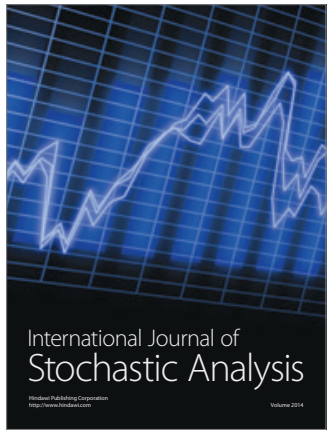

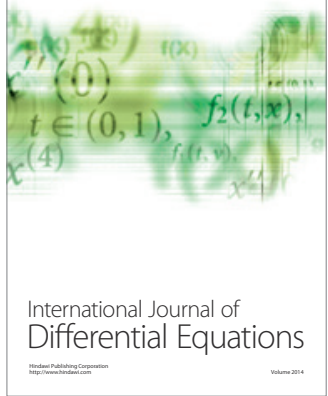
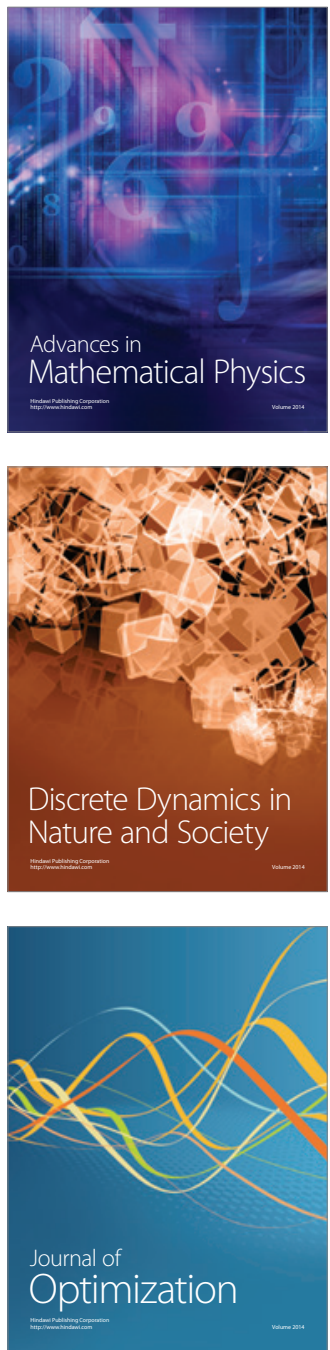